pound cholecalciferol or ergocalciferol, in that they may not require in vivo hydroxylation within the liver and kidney for activation. This may be important in the preterm infant where a maturational delay in the renal enzyme $1 \alpha$ hydroxylase pathway has been implicated ${ }^{5}$ as one of the factors associated with the complex condition of rickets of prematurity. As the metabolites have an enhanced biological activity with a shorter half life compared with the parent compounds, the dose-response relation may be more easily controlled. The routine use of these agents in the prophylaxis or management of rickets of prematurity is, however, disputed. ${ }^{6}$

This study shows, for the first time, that $1 \alpha, 25$ dihydroxycholecalciferol (Rocaltrol) is adequately absorbed after oral administration and has a similar kinetic profile to that observed in adults. ${ }^{3}$ The precise timing of the peak concentration is uncertain as frequent blood sampling was not considered ethically justifiable in these infants. The gradual and persistent rise in plasma $1 \alpha, 25$-dihydroxycholecalciferol concentration after the oral administration of One-Alpha suggests that this analogue was also absorbed and subsequently underwent liver 25-hydroxylation. Whether 25-hydroxylation is necessary for maximal biological activity of One-
Alpha is uncertain (Leo Laboratories. Personal communication).

We thank the nursing staff of the Special Care Baby Unit at Queen Charlotte's Maternity Hospital for their help and Roche Products UK and Leo Laboratories UK for providing the active agents and for financial and technical support.

\section{References \\ ${ }^{1}$ Seino Y, Ishii T, Shimotsuji T, Ishida M, Yobuuchi H. Plasma active vitamin $D$ concentration in low birthweight infants with rickets and its response to vitamin D treatment. Arch Dis Child 1981;56:628-32. \\ 2 Barek Y, Milbaer B, Weilsman Y, Edelstein S, Spirev Z. Response of neonatal hypocalcaemia to 1-alpha hydroxyvitamin $\mathrm{D}_{3}$. Arch Dis Child 1979;54:642-3. \\ 3 Mason RS, Lissner D, Posen S, Norman AW. Blood concentra- tions of dihydroxylated vitamin $D$ metabolites after an oral dose. Br Med J 1980;280:449-50. \\ ${ }^{4}$ Mallon JP, Hamilton JG, Nauss-Karol C, et al. An improved competitive protein binding assay for 1,25-dihydroxyvitamin D. Arch Biochem Biophys 1980;201:277-85. \\ ${ }^{5}$ Kovar I, Mayne P, Wallis J. Neonatal rickets in one of identical twins. Arch Dis Child 1982;57:792-4. \\ ${ }^{6}$ Brooke OG, Lucas A. Metabolic bone disease in preterm infants. Arch Dis Child 1985;60:682-5.}

Correspondence to Dr I Z Kovar, Department of Child Health, Charing Cross Hospital, Fulham Palace Road, London W6.

Received 27 February 1986

\title{
Chlamydia trachomatis as a cause of neonatal conjunctivitis
}

\author{
W C BARRY, E L TEARE, A H C UTTLEY, S A WILSON, T J MCMANUS, K S LIM, \\ H GAMSU, AND J F PRICE
}

Public Health Laboratory, Dulwich Hospital, and Departments of Genito-Urinary Medicine and Child Health, King's College Hospital, London

SUMMARY Chlamydia trachomatis was identified in 37 of 73 consecutive neonates with purulent conjunctivitis, including four delivered by caesarean section with intact membranes. Most (28/37) presented in the first week. Infection was significantly associated with referral from the community. Genital C. trachomatis infection was present in 13 of 35 parents of affected infants.

Neonatal purulent eye discharge is common. British studies in $1977^{1}$ and $1982^{2}$ and a recent Danish report $^{3}$ have given rates of $8.4 \%, 12 \%$, and $25 \%$, respectively.

Bacterial pathogens were isolated in $33 \%$ of cases in the British study of $1982^{2}$ compared with $26 \cdot 6 \%$ in an American series ${ }^{4}$ in which Chlamydia trachomatis was isolated in a further $29.5 \%$ of cases as against $3 \%$ in the 1982 British study ${ }^{2}$ and none in the 1977 study. ${ }^{1}$

Because $C$. trachomatis has been increasingly identified in the Camberwell Health Authority as a cause of pelvic inflammatory disease and nonspecific and non-gonococcal genital infection ${ }^{5}$ we have studied the pattern and causes of neonatal conjunctivitis in our area. Parents of neonates with chlamydial or gonococcal conjunctivitis were investigated for genital infection.

\section{Patients and methods}

From August 1984 to January 1985 consecutive neonates with purulent conjunctivitis were recruited from the postnatal wards of King's College and Dulwich Hospitals, the neonatal intensive care unit, 
accident and emergency department, and children's wards of King's College Hospital, and by home visits. They were seen on the day conjunctivitis was notified except for two investigated the following morning. The presence of pus, conjunctival injection, inflammation of eyelids, and periorbital oedema were recorded. Maternal age, mode of delivery, sex, race, gestation, and age at presentation of the neonate were documented. Referral of the infant from hospital or community was recorded. Pus for bacteriological investigation was collected before scraping the lower palpebral fissure with a pernasal swab. This was rolled over three $4 \mathrm{~mm}$ diameter areas of a slide, ensuring deposition of visible material, which was dried with air and fixed with acetone at the bedside. The swab tip was placed in chlamydia transport medium. Specimens were delivered and processed promptly. Techniques for immunofluorescence and isolation of $C$. trachomatis are described elsewhere. ${ }^{5}$ Slides and cultures for chlamydia were coded and examined by one observer (CW), who did not know the clinical history.

After collection of specimens babies with conjunctivitis were started on treatment with neomycin eye drops. If $C$. trachomatis was cultured treatment was changed to tetracycline eye drops and oral erythromycin for two weeks when further specimens were collected for examination for $C$. trachomatis. Parents of infants with swabs positive for $C$. trachomatis were invited to attend the genitourinary department for investigation as described elsewhere. ${ }^{5}$

\section{Results}

Seventy three neonates with conjunctivitis were studied; 60 from hospital and 13 from the community. Pathogens were identified from 44 infants $(60 \%)$. In $38(51 \%)$ the organism was $C$. trachomatis (Table). Using $C$. trachomatis culture as the standard, sensitivity of immunofluorescence was $93 \%$ and specificity $90 \%$.

Most infants with conjunctivitis presented in the first week $(\mathrm{n}=58)$, and $C$. trachomatis was identified

Table Micro-organisms identified in 73 neonates with conjunctivitis

\begin{tabular}{lc}
\hline Micro-organism & No $(\%)$ identified \\
\hline Chlamydia trachomatis & $31(42)$ \\
C. trachomatis and Staphylococcus aureus & $5(7)$ \\
C. trachomatis and Neisseria gonorrhoeae & $1(1)$ \\
S. aureus & $6(8)$ \\
Mixed coliforms & $1(1)$ \\
None & $29(40)$ \\
\hline
\end{tabular}

in $29(50 \%)$ of them. The incidence of $C$. trachomatis isolation among neonates presenting with conjunctivitis after the first week was similar, being eight out of $15(53 \%)$. The mean age at presentation was 12 days in community cases and 4 days in hospital cases $(\mathrm{p}<0.05$, Student's $t$ test). There were no differences in gestation, mode of delivery, sex, maternal age, or race between neonates with positive or negative investigations for $C$. trachomatis.

In the three months August-October 1984, 32 of 47 neonates $(68 \%)$ had swabs positive for $C$. trachomatis, whereas five of 26 neonates (19\%) recruited in the three months November 1984January 1985 had swabs positive for $C$. trachomatis $\left(p<0 \cdot 001, \chi^{2}\right.$ test $)$.

Conjunctival injection was more common in neonates with bacterial or chlamydial conjunctivitis $\left(0.05>p>0.02, \chi^{2}\right.$ test) but no more common in those with $C$. trachomatis than in those infected with other organisms. No association was found between periorbital swelling and inflammation and isolation of an organism.

Twenty six infants with chlamydial conjunctivitis were seen after treatment. Two had mild persistent discharge and $C$. trachomatis was reisolated from one. All other follow up swabs yielded negative results.

Thirty five parents of 24 infants with chlamydial conjunctivitis were investigated for genital infection. C. trachomatis was identified in 13 parents. A swab from one also grew Neisseria gonorrhoeae. Seven infants born by caesarean section had swabs positive for $C$. trachomatis. Four of these sections were performed electively before onset of labour and membrane rupture. The four parents of two of these neonates were investigated. All had $C$. trachomatis genital infection.

\section{Discussion}

In this study of neonatal conjunctivitis the most commonly isolated pathogen was $C$. trachomatis (51\%). Immunofluorescence compared favourably with culture for detection of $C$. trachomatis. The test is rapid, taking roughly 30 minutes, compared with two to three days for culture. Rapid identification of C. trachomatis before discharge of infants from hospital avoids both delay in beginning treatment and loss of patients to follow up. Chlamydial conjunctivitis was more common in patients referred from the community, presumably because the incubation period can be long (>seven days) and infants may be discharged before symptoms develop. Nevertheless, C. trachomatis was found in 27 babies (45\%) from an unselected group in hospital, some having severe conjunctivitis. 
Chlamydial conjunctivitis was diagnosed more often in autumn than winter. There was no difference in birth rate in the health authority in these seasons. Others have found $C$. trachomatis conjunctivitis to occur more often in the fourth quarter of the year. The reason for this is unknown. ${ }^{6}$

Neonates are thought to acquire $C$. trachomatis during passage through the birth canal. In our study four babies with chlamydial conjunctivitis had been delivered by caesarean section in the presence of intact membranes. Both parents of two of these babies had $C$. trachomatis genital infection. This suggests that babies may acquire $C$. trachomatis by routes other than passage through an infected cervix, possibly by ascending cervical infection. Infants developing $C$. trachomatis conjunctivitis after caesarean section might, however, be infected after birth by their parents or by indirect contact with other infected infants.

Two babies of 26 seen after treatment had persistent discharge and $C$. trachomatis was reisolated from one $(4 \%)$. In another study $12 \%$ of 34 cases had persisting $C$. trachomatis after similar treatment ${ }^{7}$ As in this study, reisolation of C. trachomatis in our patient was associated with minimal discharge. Gonococcal ophthalmia is now thought to be rare in Britain. Our single case had concurrent $C$. trachomatis infection.

We believe that investigation of conjunctivitis in neonates should include specimens for $C$. trachomatis as well as bacteria. Parents of infants with chlamydial (or gonococcal) conjunctivitis should be investigated because $C$. trachomatis is a common cause of pelvic inflammatory disease and has important public health consequences.

The authors thank Syva UK for the supply of reagents, Lederle Laboratories for financial support, and Mrs Hanni Martin for her help in the preparation of the manuscript.

\section{References \\ 1 Prentice MJ, Hutchinson GR, Taylor-Robinson D. A micro- biological study of neonatal conjuctivae and conjunctivitis. $\mathrm{Br} \mathrm{J}$ Ophthalmol 1977;61:601-7. \\ 2 Pierce JM, Ward ME, Seal DV. Ophthalmia neonatorum in the 1980s: incidence, aetiology and treatment. Br J Ophthalmol 1982;66:728-31. \\ ${ }^{3}$ Molgaard IL, Neilson PB, Kaern J. A study of the incidence of neonatal conjunctivitis and of its bacterial causes including Chlamydia trachomatis. Acta Ophthalmologica (Copenh) 1982;62:461-71. \\ 4 Stenson S, Newman R, Fedukowiczs H. Conjunctivitis in the newborn: observations on incidence, cause and prophylaxis. Ann Ophthalmol 1981;13:329-34. \\ 5 Teare EL, Sexton C, Lim F, McManus T, Uttley AHC, Hodgson J. Conventional tissue culture compared with rapid immunofluorescence for identifying Chlamydia trachomatis in specimens from patients attending a genito-urinary clinic. $\mathrm{Br} \mathrm{J}$ Vener Dis 1985;61:379-82. \\ 6 Armstrong JH, Zacarias F, Rein MF. Ophthalmia neona- torum: a chart review. Pediatrics 1976;57:884-92. \\ ${ }^{7}$ Rees E, Tair IA, Hobson D, Karayiannis P, Lee N. Persistence of chlamydial infection after treatment for neonatal conjunc- tivitis. Arch Dis Child 1981;56:193-8.}

Correspondence to Dr A H C Uttley, Public Health Laboratory, Dulwich Hospital, East Dulwich Grove, London SE2 8QF.

Received 20 March 1986

\section{Nutritional treatment of congenital heart disease}

\section{BOUGLE, M ISELIN, A KAHYAT, AND J-F DUHAMEL}

Service de Pediatrie, CHU Clémenceau, and Service de Cardiologie Infantile, CHU Côte de Nacre, Caen, France

SUMmary Twelve of 13 patients with congenital heart disease given continuous enteral nutrition displayed normal growth; cardiac function remained stable or improved in 10 in spite of the water load $(146 \pm 22 \mathrm{ml} / \mathrm{kg} /$ day $)$. This is safe treatment for malnutrition in congenital heart disease.

Growth delay is commonly observed in infants with congenital heart disease. This seems to be due to both cardiac failure and malnutrition, whatever the nature of the malformation. ${ }^{1}$ In some cases malab- sorption has been found, ${ }^{1}$ but malnutrition is mainly due to poor voluntary intake..$^{1-3}$

Poor growth delays the surgical correction of the cardiac anomaly and can impede postoperative catch up growth, due to development of pulmonary hypertension. ${ }^{1}$

Few studies have attempted, however, to correct or prevent malnutrition in congenital heart disease. ${ }^{2-4}$

\section{Patients and methods}

We studied 13 patients admitted to hospital to 